\title{
Distribuição de probabilidades para precipitação máxima diária na Bacia Hidrográfica do Rio Verde, Minas Gerais
}

\author{
Camila S. Franco ${ }^{1}$, Rosângela F. P. V. Marques ${ }^{2}$, Alisson S. Oliveira ${ }^{3}$ \& Luiz F. C. de Oliveira ${ }^{4}$
}

${ }^{1}$ DEG/UFLA. Lavras, MG. E-mail: camilasf@posgrad.ufla.br (Autor correspondente).

2 DEG/UFLA. Lavras, MG. E-mail: roeflorestal@hotmail.com

${ }^{3}$ DEG/UFLA. Lavras, MG. E-mail: alissonso@hotmail.com

${ }^{4}$ DEG/UFLA. Lavras, MG. E-mail: coutinho@deg.ufla.br

\section{Palavras-chave:}

eventos extremos

estimativa de parâmetros

inferência estatística

testes de aderência

\begin{abstract}
R E S U M O
Estimativas probabilísticas de eventos extremos são de grande relevância para o planejamento das atividades humanas. As distribuições de probabilidade possibilitam a estimativa de eventos de precipitação máxima diária anual associados às suas frequências de ocorrência. Objetivou-se, com este trabalho, identificar, dentre as distribuições Gumbel, Gama a 2 parâmetros e Generalizada de Valores Extremos (GEV), qual a mais indicada para aplicação às séries históricas de precipitação máxima diária anual na bacia hidrográfica do rio Verde, no Sul de Minas Gerais. Os parâmetros de cada distribuição foram estimados pelos métodos dos momentos, máxima verossimilhança e momentos-L. Para tal, foram empregadas, neste estudo, oito estações pluviométricas com séries históricas de, no mínimo, 20 anos de dados, distribuídas pela bacia hidrográfica. Pelos testes de adequação de Kolmogorov-Smirnov, Qui-Quadrado, Anderson Darling e Filliben, a distribuição Gumbel, estimada pelos três métodos, obteve os ajustes menos adequados. A distribuição GEV, pelo método dos momentos-L, foi a mais adequada para análise probabilística de precipitação máxima diária na Bacia Hidrográfica do Rio Verde, devendo ser priorizada para planejamentos na região.
\end{abstract}

Key words: extreme events parameter estimation statistical inference statistical tests

\section{Probability distributions for maximum daily rainfall in the watershed of Rio Verde, Minas Gerais, Brazil}

\begin{abstract}
A B S T R A C T
Probabilistic estimates of extreme events are much relevant for the planning of human activities. Thus, probability distributions enable the estimation of maximum annual daily precipitation events, associated with their respective frequencies. The aim of this study was to identify, among the Gumbel distribution, Gamma at 2 parameters and the Generalized Extreme Value (GEV), which is the most suitable to be used on historical series of annual maximum daily rainfall in the Rio Verde basin in Southern Minas Gerais. The parameters of each distribution were estimated by the methods of moments, maximum likelihood and L-moments. For this, eight rainfall stations were used with historical series of at least 20 years distributed over the watershed. By statistical tests as the Kolmogorov-Smirnov, Chi-square, Anderson Darling and Filiben test, the Gumbel distribution obtained the least appropriate adjustments. The GEV distribution by L-moments method was the most appropriate for probabilistic analysis of annual daily extreme precipitation events in the Rio Verde basin and should be prioritized for planning in the region.
\end{abstract}

\section{INTRODUÇÃO}

O registro da variabilidade temporal de registros hidrológicos possibilita estimativas probabilísticas de eventos extremos. Para tanto, foi desenvolvida a teoria de probabilidades possibilitando a estimativa de eventos hidrológicos associados a diferentes frequências de ocorrência, com base em modelos matemáticos, os quais são estruturados em parâmetros estimados pela inferência estatística (Naghettini \& Pinto, 2007).

A estimativa dos parâmetros de cada distribuição de probabilidades pode ser feita a partir de uma amostra das observações em que são feitas inferências estatísticas sobre a população, por diferentes métodos. A comparação dos modelos pode ser conduzida com base em testes de aderência não paramétricos, os quais podem informar a adequabilidade do ajuste de cada modelo à série histórica de dados observados (Casella \& Berger, 1990; Naghettini \& Pinto, 2007).

$\mathrm{Na}$ análise da precipitação máxima diária verificada no período anual, na região de Piracicaba, SP, Sansigolo (2008) utilizou o método da Máxima Verossimilhança (MV) para estimativa de parâmetros de cinco distribuições de probabilidade. $\mathrm{O}$ autor concluiu, com base nos testes de Qui-quadrado, Kolmogorov Smirnov (KS), Percentis e Quantis, que a distribuição Gumbel foi a que melhor se ajustou aos dados daquela região.

Mello \& Silva (2005) constataram, ajustando os parâmetros da distribuição Gumbel por MV e pelo Método dos Momentos 
(MM) as sete séries históricas de precipitação máxima no Alto Rio Grande, maior precisão no ajuste por MV, pelo teste de $\chi^{2}$ além de variações importantes nas equações de chuvas intensas ajustadas com base nessas estimativas e, por consequência, no hidrograma de projeto, comparando os dois métodos.

Santos et al. (2009) também destacam o bom ajuste da distribuição Gumbel para geração da equação de chuvas intensas no estado do Mato Grosso do Sul, assim como Quadros et al. (2011), em Cascavel (PR). A distribuição Gumbel proporcionou melhor ajuste também na região de Riyadh, Arábia Saudita, em estudo de Al Hassoun \& Saleh (2011) para estimativa da relação intensidade-duração-frequência de chuvas.

Em estudo semelhante, Ben-Zvi (2009) ajustou as distribuições Generalizadas de Valores Extremos (GEV), Gumbel e Log-normal a quatro séries de precipitação máxima diária anual, em Israel. Na Holanda, Overeem et al. (2008) utilizaram a distribuição GEV para diferentes tempos de duração observando poucas variações em seus parâmetros. Back (2009) utilizou a distribuição Gumbel-Chow com aderência comprovada aos dados pelo teste de Kolmogorov-Smirnov, para avaliar precipitações intensas de diferentes durações no município de Urussanga, SC, no período de 1981 a 2004.

A distribuição Gama, estimada pelo método MV para precipitação mensal no Sudoeste da Bahia, obteve bom ajuste em duas série históricas, segundo o teste de KS por Murta et al. (2005). Beijo et al. (2009) observaram bom ajuste da distribuição GEV estimada pelo método da MV aplicada em eventos de precipitação máxima na região de Jaboticabal, SP.

Como a distribuição GEV é destacada em muitos estudos para estimativa de eventos extremos, Queiroz \& Chaudhry (2006) apresentam sua modelagem em Matlab utilizando o método dos momentos L para estimativa dos parâmetros. Com base nos testes de Wang e KS, os autores consideraram o modelo adequado a 5\% de probabilidade. Na Bacia do La Plata, Naumann et al. (2012) destacaram melhor ajuste das distribuições GEV e Gama aos dados observados.

Em destaque à mesma distribuição, Blain \& Moraes (2011) verificaram o ajuste da distribuição GEV em oito séries de máximos diários de precipitação da Secretaria de Agricultura e Abastecimento do Estado de São Paulo. Os testes de aderência indicaram bom ajuste da distribuição pelo método MV. Em 120 anos de registros de precipitação máxima diária em Campinas, Blain (2011) também observou melhor adequação da distribuição GEV pelo método MV em comparação com o método ML.

Neste contexto objetivou-se analisar a precisão dos ajustes de três distribuições de probabilidade (Gumbel, Gama e GEV) utilizando, para estimativa dos parâmetros, os métodos dos momentos (MM), Máxima Verossimilhança (MV) e Momentos L (ML), em oito séries históricas de precipitação máxima diária anual, da Bacia Hidrográfica do Rio Verde a fim de identificar a distribuição e o melhor método que se aplicam para esta importante região de Minas Gerais.

\section{Material e Métodos}

A Bacia Hidrográfica do rio Verde corresponde a 4,25\% da área total da Bacia Hidrográfica do Rio Grande, constituindo a Unidade de Planejamento e Gestão dos Recursos Hídricos UPGRH GD4, na região Sul de Minas Gerais (CBH Grande, 2012). Possui área de drenagem de $6.891,4 \mathrm{~km}^{2}$, onde estão inseridos 31 municípios com população total de cerca de 450 mil habitantes.

O clima da região é mesotérmico brando e úmido, com 3 meses secos e temperatura média anual de 18 a $19{ }^{\circ} \mathrm{C}$, ou seja, do tipo Cwa em sua grande maioria, podendo ocorrer $\mathrm{Cwb}$ na região de cabeceira. $\mathrm{O}$ alto curso do rio Verde se localiza próximo ao município de São Lourenço, onde predominam encostas de grande declividade e solos rasos. A região de Varginha corresponde ao baixo rio Verde, com altitudes variando entre 900 e $1.000 \mathrm{~m}$ (CBH Grande, 2012).

Junto ao Sistema de Informações Hidrológicas da Agência Nacional das Águas, ANA (2012) foram selecionadas oito estações pluviométricas (Tabela 1) distribuídas pela sub-bacia do Rio Verde (Figura 1).

Para cada série histórica de dados de chuvas extremas foram ajustados os parâmetros das distribuições de Gumbel,

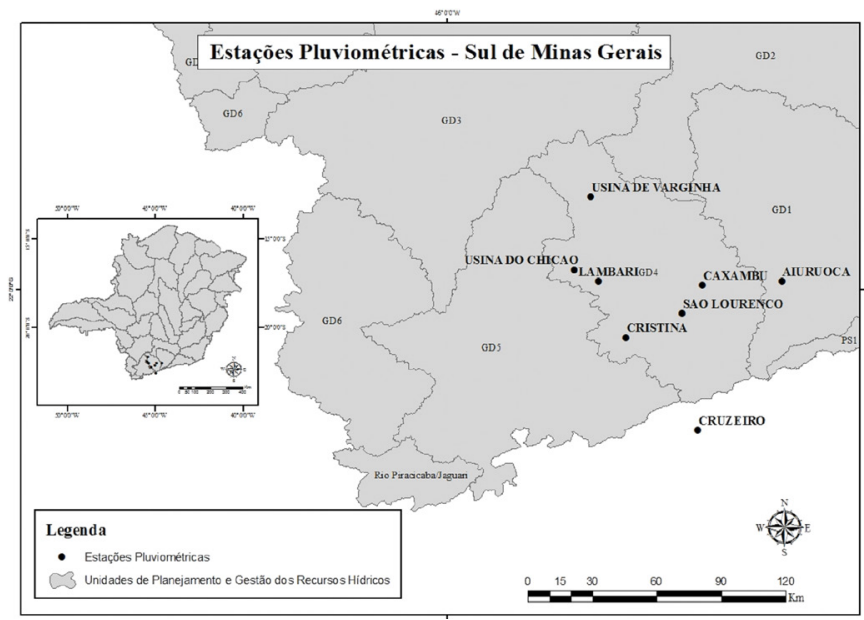

Figura 1. Distribuição das estações pluviométricas na região da Sub-Bacia do Rio Verde

Tabela 1. Estações pluviométricas empregadas

\begin{tabular}{lcccc}
\hline Nome da estação & Código & Município & Número de dados da série histórica & Período da série histórica \\
Aiuruoca & 2144018 & Aiuruoca & 60 & 1948 a 2007 \\
Caxambu & 2144003 & Caxambu & 60 & 1945 a 2004 \\
Cristina & 2245065 & Cristina & 60 & 1948 a 2007 \\
Cruzeiro & 2244003 & Cruzeiro & 58 & 1934 a 1995 \\
São Lourenço & 2245107 & São Lourenço & 38 & 1968 a 2008 \\
Usina de Varginha & 2145018 & Varginha & 30 & 1943 a 1978 \\
Usina do Chicão & 2145009 & Campanha & 57 & 1942 a 2006 \\
Lambari & 2145013 & Lambari & 20 & 1942 a 1961 \\
\hline
\end{tabular}


Generalizada de Valores Extremos (GEV) e Gama 2 parâmetros cujas funções de densidade de probabilidade (FDP) são dadas pelas Eqs. 1, 2 e 3, respectivamente:

$$
\begin{gathered}
\text { FDP }=\alpha \cdot \mathrm{e}^{\left\{-\alpha(\mathrm{x}-\mu)-\mathrm{e}^{-\alpha(\mathrm{x}-\mu)}\right\}} \\
\mathrm{FDP}=\mathrm{f}(\mathrm{x})=\frac{1}{\alpha} \cdot\left[1-\xi \cdot\left(\frac{\mathrm{x}-\mu}{\alpha}\right)\right]^{\left(\frac{1+\xi}{\xi}\right)} \cdot \exp \left\{-\left[1-\xi \cdot\left(\frac{\mathrm{x}-\mu}{\alpha}\right)\right]^{\frac{1}{\xi}}\right\} \\
\text { FDP:f }(\mathrm{x})=\frac{1}{\beta^{\mathrm{v}} \times \Gamma(\mathrm{v})} \cdot \mathrm{x}^{\mathrm{v}-1} \cdot \mathrm{e}^{\frac{-\mathrm{x}}{\beta}}
\end{gathered}
$$

em que:

X - precipitação máxima diária anual

$\alpha, \mu, \beta, v$ e $\xi$ - parâmetros da distribuição de probabilidade

Foram empregados, na estimativa dos parâmetros das distribuições de probabilidade, os métodos dos momentos (MM), máxima verossimilhança (MV) e momentos $\mathrm{L}$ (ML). Portanto, foram ajustados, para cada distribuição de probabilidade, seus parâmetros pelos métodos de estimativas. Para detalhes sobre as citadas metodologias, recomenda-se Naghettini \& Pinto (2007), Casella \& Berger (1990) e Haan (2002)

Para verificação da aderência dos ajustes dos parâmetros das distribuições de probabilidade, foram empregados os testes de Kolmogorov Smirnov $(\Delta \mathrm{F})$, Qui-Quadrado $\left(\chi^{2}\right)$, Anderson Darling $\left(\mathrm{AD}^{2}\right)$ e Filliben $\left(\mathrm{r}_{\text {calc }}\right)$.

$\mathrm{Na}$ obtenção do $\Delta \mathrm{F}$, calculou-se o erro absoluto máximo entre a frequência observada e a probabilidade estimada. $\mathrm{O}$ valor de $\Delta \mathrm{F}$ tabelado é encontrado em função da significância de $5 \%$ e do número de dados da série histórica. Para valores menores que os tabelados o modelo foi classificado adequado (Eq. 4).

$$
|\Delta \mathrm{F}|_{\text {calculadomáx }} \leq|\Delta \mathrm{F}|_{\text {tabela }(\mathrm{n}, \alpha)}
$$

Para o cálculo de $\chi^{2}$ foram geradas classes de frequência para a série de dados das quais foram extraídas as teóricas com cada distribuição por cada método de ajuste de parâmetros. $\mathrm{O}$ somatório das diferenças quadráticas (Eq. 5) resulta no valor de $\chi^{2}$ calculado, para valores menores que o $\chi^{2}$ tabelado (em função do grau de significância e do grau de liberdade) o modelo foi classificado adequado.

$$
\chi_{\text {calculado }}^{2}=\sum_{\mathrm{i}=1}^{\mathrm{n}} \frac{\left(\mathrm{fo}_{\mathrm{i}}-\mathrm{ft}_{\mathrm{i}}\right)^{2}}{\mathrm{ft}_{\mathrm{i}}}
$$

em que:

fo e $\mathrm{ft}$ - frequência observada e calculada para cada intervalo, respectivamente

$\mathrm{O}$ teste de aderência de $\mathrm{AD}^{2}$ baseia-se na diferença entre as funções de probabilidade empírica e teórica das variáveis dando mais peso às caudas das distribuições cuja estatística é equivalente à Eq. 6 (Naghettini \& Pinto, 2007).

$\mathrm{AD}^{2}=-\mathrm{N}-\frac{\left[\sum(2 \cdot \mathrm{i}-1) \cdot\left[\left(\mathrm{LN}\left(\mathrm{P}_{1}(\mathrm{X}<\mathrm{xi})\right)\right)+\left(\mathrm{LN}\left(\mathrm{P}_{2}(\mathrm{X}>\mathrm{xi})\right)\right)\right]\right]}{\mathrm{N}}$

em que:

i - ordem dos dados da série histórica

$\mathrm{P}_{1}(\mathrm{X}<\mathrm{xi})$ - probabilidade de não excedência

$\mathrm{P}_{2}(\mathrm{X}>\mathrm{xi})$ - probabilidade de excedência e $\mathrm{N}$ o número de dados

Para valores de $\mathrm{AD}^{2}$ elevados as funções diferem muito e a adequabilidade do modelo é rejeitada. Para valores menores que os tabelados (dependentes da distribuição e do grau de significânicia), os modelos foram classificados adequados.

O teste de aderência de Filliben estima um coeficiente de correlação $\left(\mathrm{r}_{\text {calc }}\right)$ entre as observações $\mathrm{x}_{\mathrm{i}}$ e os quantis teóricos $\mathrm{w}_{\mathrm{i},}$ calculados pela Eq. 7 .

$$
\mathrm{wi}=\mathrm{F}^{-1}(\mathrm{qi})
$$

em que:

$\mathrm{F}^{-1}$ - função inversa da FDP e $\mathrm{q}_{\mathrm{i}}$ a frequência observada, calculada pela Eq. 8

$$
\mathrm{qi}=\frac{\mathrm{i}-0,4}{\mathrm{~N}+1-2 \cdot 0,4}
$$

em que:

$\mathrm{N} \quad$ - número de dados da série histórica

i - posição ocupada pelo valor da série

O coeficiente de correlação entre $\mathrm{x}_{\mathrm{i}}$ e $\mathrm{w}_{\mathrm{i}}$ é então calculado pela Eq. 9.

$$
r_{\text {calc }}=\frac{\sum_{i=1}^{N}\left(x_{i}-\bar{x}\right) \cdot\left(w_{i}-\bar{w}\right)}{\sqrt{\sum_{i=1}^{N}\left(x_{i}-\bar{x}\right)^{2} \cdot \sum_{i=1}^{N}\left(w_{i}-\bar{w}\right)^{2}}}
$$

Para valores de $\mathrm{r}_{\text {calc }}$ maiores que os tabelados (em função da distribuição, do número de dados da série histórica e do grau de significância) o modelo é classificado adequado.

\section{Resultados e Discussão}

Para uma mesma distribuição de probabilidade foi possível observar variações nos ajustes dos parâmetros ao se utilizar os diferentes métodos de estimativa; foram observadas, também, variações nos ajustes entre diferentes distribuições para um mesmo método de estimativa de parâmetros.

A Figura 2 apresenta, a titulo de exemplo, os ajustes da distribuição Gumbel, GEV e Gama para a série histórica de Aiuruoca, cujos parâmetros foram estimados pelos três métodos 
A.

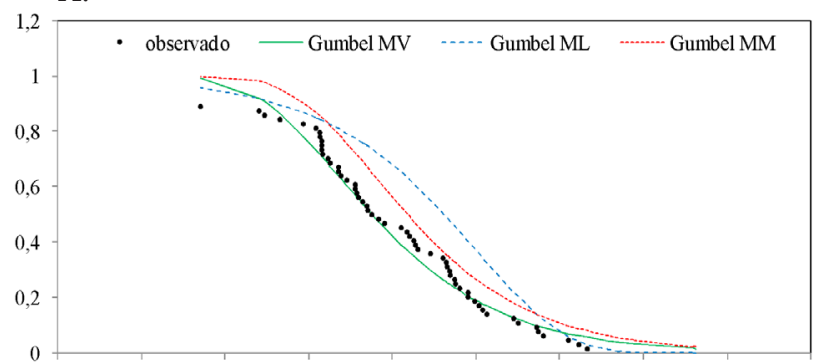

B.

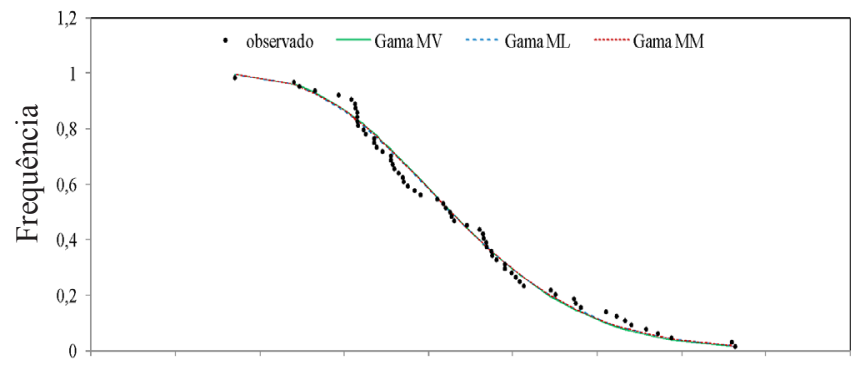

C.

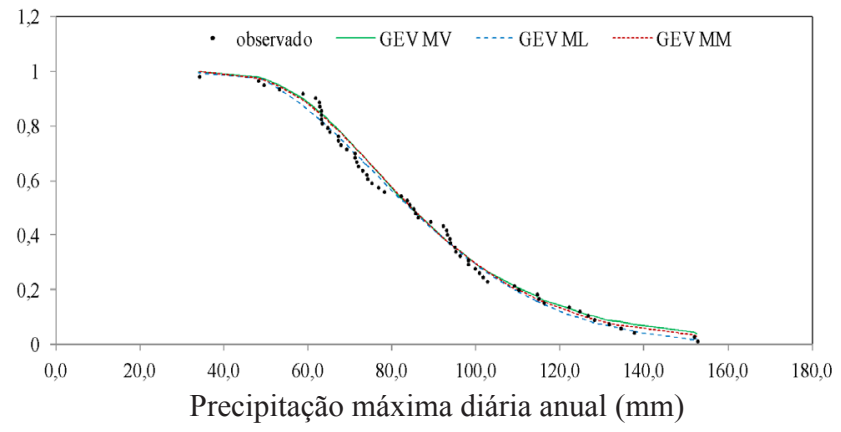

Figura 2. Distribuição de frequência à série histórica de precipitação máxima diária anual da estação de Aiuruoca, MG pelas distribuições de probabilidade (A) distribuição de Gumbel, (B) distribuição Gama e (C) distribuição GEV empregando-se os diferentes métodos de estimativa dos parâmetros empregados neste estudo; para as demais séries históricas os ajustes se apresentaram de forma semelhante.

Verificaram-se, nos ajustes da distribuição Gumbel, diferenças entre os métodos de estimativa sendo que o melhor ajuste aos valores médios foi obtido pelo método da MV enquanto o método dos ML se ajustou melhor a valores mínimos. Para esta distribuição a estimativa dos parâmetros pelo método do MM foi a que obteve os maiores desvios entre as frequências calculada e teórica (Figura 2A). Observaram-se comportamentos semelhantes para as demais séries históricas.

Constata-se, na referida figura, que os melhores ajustes foram obtidos para as distribuições GEV e Gama (Figura 2B e 2C) principalmente os valores máximos e mínimos. Nessas duas distribuições percebeu-se grande semelhança nos ajustes dos três métodos de estimativa de parâmetros.

A fim de se analisar estatisticamente os ajustes identificandose a melhor distribuição de probabilidade possível e o método de estimativa de parâmetros para a bacia do Rio Verde, a Tabela 2 apresenta os resultados dos testes de aderência para cada distribuição e série trabalhadas.

Dentre os quatro testes de aderência empregados neste estudo foi possível observar uma restrição maior gerada pelos testes de Anderson Darling e Filliben. O primeiro teste é interessante por analisar o comportamento dos ajustes atribuindo maior peso aos valores das caudas das distribuições, o que é extremamente relevante para dados com característica assintótica, tais como os de precipitação máxima diária anual.

Entre os testes de Filliben, o de aderência foi o mais rigoroso sendo que em 51,39\% das análises as distribuições foram consideradas inadequadas. A distribuição Gama foi a mais afetada seguida das GEV e Gumbel. Por outro lado, o teste de Anderson-Darling classificou inadequadas $43,06 \%$ das distribuições, principalmente a de Gumbel.

Os testes de Qui-quadrado e Kolmogorov-Smirnov produziram um número maior de adequações; em apenas

Tabela 2. Adequabilidade das distribuições de probabilidades para as séries históricas de precipitação máxima diária anual, para a Bacia do Rio Verde, Minas Gerais

\begin{tabular}{|c|c|c|c|c|c|c|}
\hline \multirow{2}{*}{ Estação } & \multirow{2}{*}{ Distribuição } & \multirow{2}{*}{ Método } & \multicolumn{4}{|c|}{ Teste de Aderência } \\
\hline & & & KS & $x^{2}$ & $\overline{A D^{2}}$ & Filliben \\
\hline \multirow{5}{*}{ Aiuruoca } & Gumbel & MV & Adequado & Inadequado & Inadequado & Adequado \\
\hline & \multirow{3}{*}{ GEV } & MM & Adequado & Adequado & Adequado & Adequado \\
\hline & & MV & Adequado & Adequado & Adequado & Adequado \\
\hline & & $\mathrm{ML}$ & Adequado & Adequado & Adequado & Adequado \\
\hline & Gama 2P & $M L$ & Adequado & Adequado & Adequado & Inadequado \\
\hline \multirow[b]{5}{*}{ Usina do Chicão (Campanha) } & \multirow{3}{*}{ Gumbel } & MM & Adequado & Inadequado & Inadequado & Adequado \\
\hline & & MV & Adequado & Inadequado & Inadequado & Adequado \\
\hline & & ML & Adequado & Inadequado & Inadequado & Inadequado \\
\hline & \multirow[b]{2}{*}{ GEV } & MM & Adequado & Adequado & Inadequado & Inadequado \\
\hline & & MV & Adequado & Inadequado & Inadequado & Inadequado \\
\hline
\end{tabular}


Continuação da Tabela 2

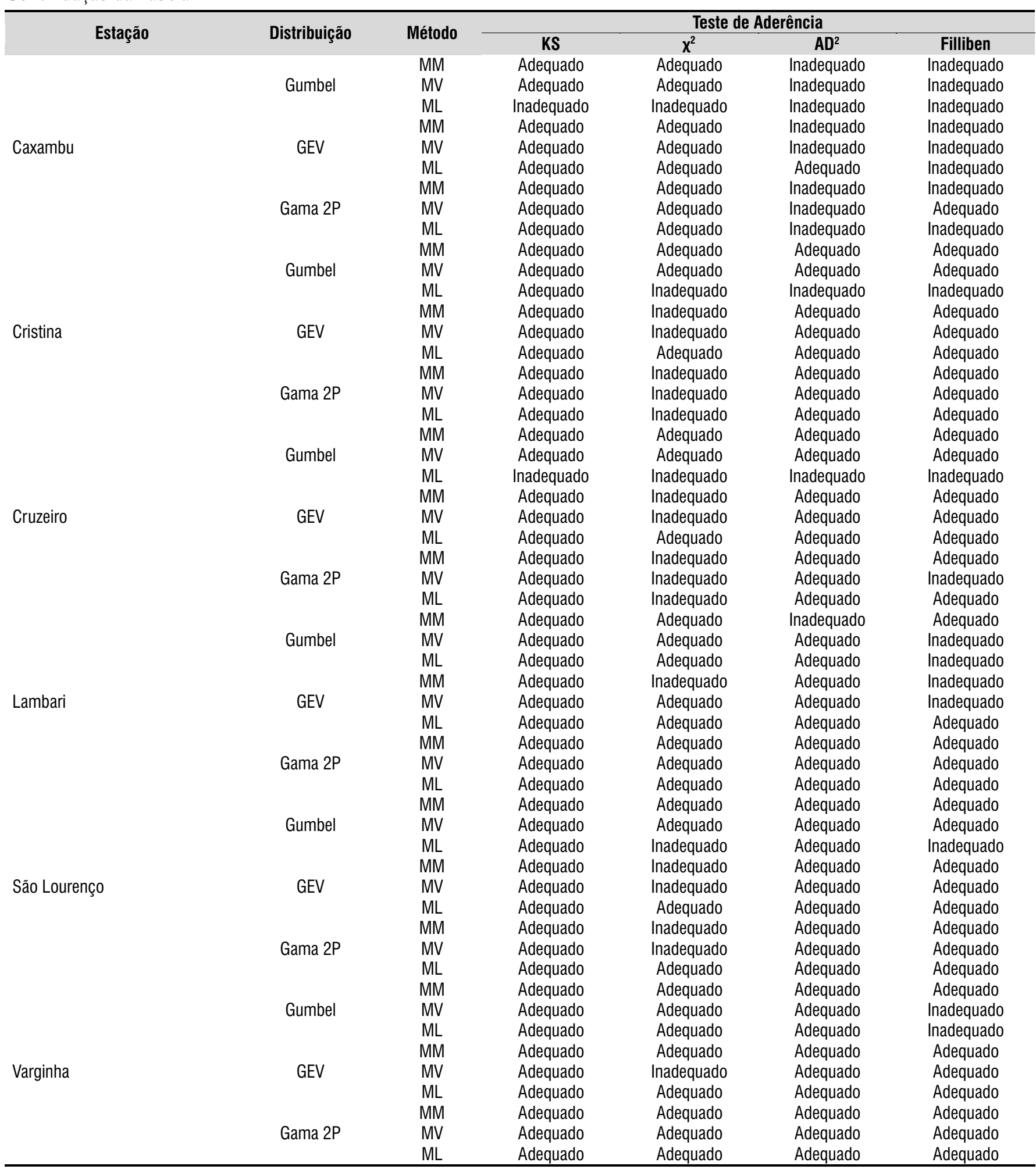

$18,06 \%$ das situações as distribuições foram classificadas inadequadas com alta concentração na distribuição Gumbel. Pelos resultados obtidos verifica-se a fragilidade desses testes para a análise de séries históricas assintóticas e devem ser evitados para reduzir erros do tipo I, ou seja, aceitar a hipótese Ho de que a distribuição é adequada sendo que a mesma pode não representar adequadamente a população de dados.
Dos três métodos de estimativa de parâmetros empregados neste estudo, o método dos ML obteve o maior número de inadequações (36\%), seguido do método da MV (34\%) e o MM (30\%). No entanto, no estudo de Murta et al. (2005) para precipitações mensais aplicando esta mesma distribuição, os autores destacaram o método MV como mais adequado para estimativa dos parâmetros embora não tenham testado o método ML. 
Para a distribuição de Gumbel o MM foi o mais adequado enquanto que em outros estudos, como os de Sansigolo (2008) e Mello \& Silva (2005) o método MV foi o mais adequado para esta distribuição. Para os três métodos a distribuição Gumbel obteve o menor número de adequações nas séries históricas estudadas, ao contrário do observado nos estudos de Santos et al. (2009), Quadros et al. (2011) e Al Hassoun \& Saleh (2011).

Dentre as três distribuições estimadas pelos três métodos (MM, MV e ML) a GEV, obteve, com base no método ML, maior número de adequações e, portanto, o melhor ajuste às series de dados para a bacia do rio Verde significando melhores estimativas de eventos extremos diários para a referida bacia concordando com os resultados obtidos em outros estudos no Brasil, tais como Blain (2011) e Beijo et al. (2009).

Como os testes de $\mathrm{AD}^{2}$ e de Filliben foram considerados os mais restritivos, as Tabelas 3 e 4 apresentam os valores

Tabela 3. Precisão do teste de Anderson-Darling nas distribuições adequadas de probabilidades para as séries históricas de precipitação máxima diária anual para a Bacia do Rio Verde, Minas Gerais

\begin{tabular}{|c|c|c|}
\hline Estação & Modelo & $\begin{array}{c}\left.\mathrm{AD}^{2} \text { (Tabelado }=0,757\right) \\
\text { Calculado }\end{array}$ \\
\hline \multirow{4}{*}{ Aiuruoca } & GEV MM & 0,354 \\
\hline & GEV MV & 0,327 \\
\hline & GEV ML & 0,219 \\
\hline & Gama ML & 0,384 \\
\hline Usina do Chicão (Campanha) & GEV ML & 0,733 \\
\hline \multirow[t]{4}{*}{ Caxambu } & GEV ML & 0,710 \\
\hline & Gumbel MM & 0,675 \\
\hline & Gumbel MV & 0,028 \\
\hline & GEV MM & 0,664 \\
\hline \multirow{8}{*}{ Cristina } & GEV MV & 0,671 \\
\hline & GEV ML & 0,676 \\
\hline & Gama MM & 0,650 \\
\hline & Gama MV & 0,701 \\
\hline & Gama ML & 0,687 \\
\hline & Gumbel MM & 0,497 \\
\hline & Gumbel MV & 0,330 \\
\hline & GEV MM & 0,498 \\
\hline \multirow[t]{6}{*}{ Cruzeiro } & GEV MV & 0,692 \\
\hline & GEV ML & 0,187 \\
\hline & Gama MM & 0,724 \\
\hline & Gama ML & 0,745 \\
\hline & Gumbel MM & 0,613 \\
\hline & GEV ML & 0,218 \\
\hline \multirow[t]{6}{*}{ Lambari } & Gama MM & 0,423 \\
\hline & Gama MV & 0,414 \\
\hline & Gama ML & 0,415 \\
\hline & Gumbel MM & 0,419 \\
\hline & Gumbel MV & 0,404 \\
\hline & GEV MM & 0,386 \\
\hline \multirow{7}{*}{ São Lourenço } & GEV MV & 0,407 \\
\hline & GEV ML & 0,330 \\
\hline & Gama MM & 0,328 \\
\hline & Gama MV & 0,328 \\
\hline & Gama ML & 0,323 \\
\hline & Gumbel MM & 0,534 \\
\hline & GEV MM & 0,615 \\
\hline \multirow{4}{*}{ Varginha } & GEV MV & 0,753 \\
\hline & GEV ML & 0,325 \\
\hline & Gama MM & 0,466 \\
\hline & Gama MV & 0,448 \\
\hline
\end{tabular}

Tabela 4. Precisão do teste de Filliben nas distribuições adequadas de probabilidades para as séries históricas de precipitação máxima diária anual, para a Bacia do Rio Verde, Minas Gerais

\begin{tabular}{|c|c|c|c|}
\hline \multirow{2}{*}{ Estação } & \multirow{2}{*}{ Modelo } & \multicolumn{2}{|c|}{ Filliben } \\
\hline & & Calculado & Tabelado \\
\hline \multirow{6}{*}{ Aiuruoca } & Gumbel MM & 0,989 & \multirow{6}{*}{0,969} \\
\hline & Gumbel MV & 0,989 & \\
\hline & GEV MM & 0,989 & \\
\hline & GEV MV & 0,983 & \\
\hline & GEV ML & 0,994 & \\
\hline & Gumbel MM & 0,976 & \\
\hline \multirow[t]{2}{*}{ Usina do Chicão (Campanha) } & Gumbel MV & 0,985 & \multirow[t]{2}{*}{0,969} \\
\hline & GEV ML & 0,979 & \\
\hline \multirow[t]{2}{*}{ Caxambu } & Gama MV & 0,999 & \multirow[t]{2}{*}{0,969} \\
\hline & Gumbel MM & 0,992 & \\
\hline \multirow[t]{3}{*}{ Cristina } & Gumbel MV & 0,993 & \multirow[t]{3}{*}{0,972} \\
\hline & GEV ML & 0,991 & \\
\hline & Gumbel MM & 0,984 & \\
\hline \multirow[t]{5}{*}{ Cruzeiro } & Gumbel MV & 0,982 & \multirow[t]{2}{*}{0,972} \\
\hline & GEV ML & 0,979 & \\
\hline & Gumbel MM & 0,960 & \multirow{8}{*}{0,939} \\
\hline & Gumbel MV & 0,955 & \\
\hline & Gumbel ML & 0,977 & \\
\hline \multirow{6}{*}{ Lambari } & GEV MV & 0,944 & \\
\hline & GEV ML & 0,954 & \\
\hline & Gama MM & 0,990 & \\
\hline & Gama MV & 0,990 & \\
\hline & Gama ML & 0,990 & \\
\hline & Gumbel MM & 0,987 & \multirow{4}{*}{0,969} \\
\hline \multirow{6}{*}{ São Lourenço } & Gumbel MV & 0,996 & \\
\hline & GEV ML & 0,980 & \\
\hline & Gama ML & 0,973 & \\
\hline & Gumbel MM & 0,961 & \multirow{8}{*}{0,953} \\
\hline & Gumbel MV & 0,970 & \\
\hline & Gumbel ML & 0,953 & \\
\hline \multirow{5}{*}{ Varginha } & GEV MM & 0,954 & \\
\hline & GEV ML & 0,987 & \\
\hline & Gama MM & 0,976 & \\
\hline & Gama MV & 0,981 & \\
\hline & Gama ML & 0,975 & \\
\hline
\end{tabular}

calculados e tabelados dos modelos que foram classificados adequados pelos testes, para melhor analisar a precisão de cada modelo.

Pelos resultados do teste de $\mathrm{AD}^{2}$, a distribuição GEV estimada pelo método dos ML obteve maior número de menores valores calculados nas estações analisadas (75\%); entretanto, pelo teste de Filliben, o maior número de maiores valores calculados corresponde à distribuição Gumbel calculada pelo método da MV (37\%).

Pode-se dizer, para a Bacia do Rio Verde, que a distribuição GEV pelo método ML e a distribuição Gumbel pelo método MV são as mais adequadas para estudos de probabilidades de precipitação máxima diária anual. Como consequência, em estudos de chuvas intensas e de projeto e planejamento de obras hidráulicas é coerente a utilização desses modelos.

\section{Conclusões}

1. Dos testes de aderência KS, $\chi^{2}, \mathrm{AD}^{2}$ e Filliben aplicados às séries de dados históricos de precipitação 
máxima anual, o mais restritivo foi o de Filliben, seguido pelo de $\mathrm{AD}^{2}$.

2. Dentre os nove ajustes em cada uma das oito séries de dados históricos distribuídos na região da Sub-Bacia do Rio Verde, a distribuição de probabilidades GEV utilizando o método dos ML para estimativa dos parâmetros e a distribuição Gumbel pelo método $\mathrm{MV}$, foram consideradas as mais adequadas para estudos de probabilidade de precipitação máxima diária anual.

\section{Literatura Citada}

Al Hassoun; Saleh, A. Developing an empirical formulae to estimate rainfall intensity in Riyadh region. Journal of King Saud University, v.23, p.81-88, 2011

ANA - Agência Nacional das Águas. Sistema de Informações Hidrológicas. http://hidroweb.ana.gov.br/. 29 Mai. 2012.

Back, A. J. Relações entre precipitações intensas de diferentes durações ocorridas no município de Urussanga, SC. Revista Brasileira de Engenharia Agrícola e Ambiental, v.13, p.170175, 2009.

Beijo, L. A.; Vivanco, M. J. F.; Muniz, J. A. Análise Bayesiana no estudo do tempo de retorno das precipitações máximas em Jaboticabal (SP). Ciência e Agrotecnologia, v.33, p.261270, 2009.

Ben-Zvi, A. Rainfall intensity-duration-frequency relationships derived from large partial duration series. Journal of Hydrology, v.367, p.104-114, 2009.

Blain, C. G. Cento e vinte anos de totais extremos de precipitação pluvial máxima diária em Campinas, Estado de São Paulo: Análises estatísticas. Agrometeorologia, v.70, p.722-728, 2011.

Blain, C. G.; Moraes, O. S. Caracterização estatística de oito séries de precipitação pluvial máxima diária da secretaria de agricultura e abastecimento do estado de São Paulo. Revista Brasileira de Meteorologia, v.26, 255-234, 2011.
Casella, G.; Berger, R. L. Statistical inference. Wadsworth and Brooks/Cole: Pacific Grove, 1990. 650p.

CBH Grande - Comitê da Bacia Hidrográfica do Rio Grande. http://www.grande.cbh.gov.br/GD4. 16 Abr. 2012.

Haan, C. T. Statistical methods in hydrology. 2.ed. Ames: The Iowa State University, 2002. 377p.

Mello, C. R.; Silva, A. M. Métodos estimadores dos parâmetros da distribuição de Gumbel e sua influência em estudos hidrológicos de projeto. Irriga, v.10, p.318-334, 2005.

Murta, R. M.; Teodoro, S. M.; Bonomo, P.; Chaves, M. A. Precipitação pluvial mensal em níveis de probabilidade pela distribuição Gama para duas localidades do Sudoeste da Bahia. Ciência e Agrotecnologia, v.33, p.261-270, 2005.

Naghettini, M.; Pinto, E. J. A. Hidrologia estatística. Belo Horizonte: CPRM, 2007. 552p.

Naumann, g.; Llano, M. P.; Vargas, W. M. Climatology of the annual maximum daily precipitation in the La Plata Basin. International Journal of Climatology, v.32, p.247-260, 2012.

Overeem, A.; Buishand, A.; Holleman, I. Rainfall depthduration-frequency curves and their uncertainties. Journal of Hydrology, v.348, p.124-134, 2008.

Quadros, L. E.; Queiroz, M. M. F.; Boas M. A. V. Distribuição de frequência e temporal de chuvas intensas. Acta. Scientiarum. Agronomy, v.33, p.401-410, 2011.

Queiroz, M. M. F.; Chaudhry, F. H. Análise de eventos hidrológicos extremos, usando-se a distribuição GEV e momentos LH. Revista Brasileira de Engenharia Agrícola e Ambiental, v.10, p.381-389, 2006.

Sansigolo, C. A. Distribuição de extremos de precipitação diária, temperatura máxima e mínima e velocidade do vento em Piracicaba, SP (1917-2006). Revista Brasileira de Meteorologia, v.23, p.341-246, 2008.

Santos, G. G.; Figueiredo, C. C.; Oliveira, L. F. C.; Griebeler, N. P. Intensidade-duração-frequência de chuvas para o Estado de Mato Grosso do Sul. Revista Brasileira de Engenharia Agrícola e Ambiental, v.13, p.899-905, 2009. 